\title{
Aesthetic Management in Leprosy
}

\author{
TIO (TIONG-HOO) \\ National Institute of Health, Surabaya (Indonesia)* \\ 'Leprosy is dreaded most of all diseases not because it kills, but because it leaves alive'. (MUIR)
}

No truer words could have been written on leprosy than Muir's. It is well-known that leprosy patients suffer much more from mental depression rather than physical disabilities, and in his practice the writer has spent more time, especially during the initial consultation, comforting such patients rather than on drug and auxiliary treatment. The mental sufferings of leprosy patients can roughly be divided into two basic categories:

(a) knowledge of having a supposedly incurable disease which is thought to result in horrible disfiguration.

(b) constant fear that other people discover their disease and treat them as outcasts.

With regard to the former type of agony a clear explanation of the curability of the disease will boost the patients' morale. Although it is not easy to give it is far more difficult to soothe the patients' fear of becoming outcasts. It is the impression of the writer that this constant terror even overshadows the fear for the horrible somatic consequences. Visible symptoms of leprosy are dreaded, not so much for their unaesthetic appearance but rather because these symptoms will reveal the disease, so the writer feels that aesthetic management of leprosy is as important as the sulphone therapy, especially in the early stages of the disease, when the patient has to overcome his/her initial anxiety. This treatment seems to be appropriate for more well-to-do and intelligent people but the writer has experienced that the idea can also be applied to the poor. In a leprosarium, after undergoing two weeks of successful treatment for chronic plantar ulcers ${ }^{2}$, the poor inmates generally wanted to know whether the writer could also restore lost eyebrows.

The visual symptoms on exposed areas, especially on the face, which may betray the patients are:

I. thickening of or noduli in the earlobes.

2. E.N.L., leproma and leprides.
3. erythematous maculae, sometimes swollen. 4. diffuse swelling of the face.

5. madarosis, mainly of the lateral part.

6. swollen digits.

7. dull, dry, scaling, hyperpigmented skin of extremities, especially of the legs.

8. elephantiasis.

All these symptoms are caused by infiltration, granulomata and/or widening of capillaries or as a result of trophic disturbance. They may occur as short attacks or persistently for long periods and can even become permanent. The writer has the impression that it is of paramount importance to treat these symptoms as early as possible not only for psychological reasons but also to curb further and possible irreparable damage. The earlier the therapy is started the quicker the results are obtained and the least psychic damage the disease can inflict. In coloured people every inflammation, especially if of long standing, may cause hyperpigmentation. ${ }^{3}$

Many of these symptoms can be improved with diathermic sparking ${ }^{4}$ but similar results can be obtained by subcutaneous injections of preferable soluble corticosteroids. Local corticosteroid injections are very convenient and in many cases give much quicker and better aesthetic results than diathermic sparking but are far more expensive and therefore less suitable for mass campaigns. On the other hand diathermic sparking has, next to causing pain, the disadvantage that more time is required for the symptoms to disappear. Besides inflicting psychological harm the longer period before success is evident increases in coloured people the possibility of hyperpigmented sequels. Diathermic sparking itself may also give additional thermic stimulation to pigmentation. As such it is advisable to combine all diathermic sparking

* Present Address: i 8 Johan van Oldenbarneveltlaan, the Hague (Netherland).

Aesthetic Management in Leprosy

245 
for aesthetic purposes with topical bleaching agents. While in fresh eruptions bleaching agents are generally not necessary, the need for them becomes all the more pressing in lesions of long duration. In sparsely disseminated lesions, especially on the face, local subcutaneous injections of corticosteroids are preferable to the much more easily administrable oral ones because of the latter's weakening effect on the general def ence mechanism. Injections are also of great value when reaction-symptoms fail to respond to normal doses of oral corticosteroids (see case I $7 / 64$ ).

Although no wide experience has yet been gained, preliminary comparative results are herewith discussed with both methods in the aesthetic management of leprosy.

\section{Thickening of or noduli in earlobes}

Superiority of local corticosteroid injections over diathermic sparking was clear. Sometimes even one injection of $5 \mathrm{mg}$. prednisolone, especially in fresh cases, was sufficient to normalize the size of the thickened earlobe.

\section{E.N.L., Leproma and Leprides}

Although remote action of injected corticosteroids is known to exist (see under swelling of the face), it is, in the experience of the writer, by far the best method to inject all ENL lesions and lepromata separately with very small adequate quantities of corticosteroids. Complete flattening can be obtained very quickly leaving only hyperpigmented spots in more chronic cases. Recurrence of ENL may happen at short notice but in many cases this treatment gives long intermissions. Diathermic sparking reduces ENL at slower pace and with greater chances of hyperpigmentation.

In one L-type patient (17/64) with body weight of $33 \mathrm{~kg}$. only, reaction symptoms flared up on 3 tablets of $5 \mathrm{mg}$. prednisolone per day. One series of injections of in all $25 \mathrm{mg}$. cortisone under the lesions at several places of the face cleared all symptoms within a week.

In one patient (G.S.T.) with persistent uniformly raised leprides on the face, however, repeated subcutaneous cortisone injections and oral prednisone could not reduce the plagues. Yet diathermic sparking had some softening and reducing effect.

246 Leprosy Review

\section{Erythematous maculae}

In coloured people, any chronic erythema if not treated may result in hyperpigmentation and bleaching agents are a necessity in the treatment of erythematous maculae of long duration. Subtcuaneous corticosteroids have a quick beneficial effect.

Early injections are desirable as demonstrated in one case of extensive erythema of one month on both cheeks for which the patient was expelled from her house. One series of subcutaneous injections of in all $25 \mathrm{mg}$. cortisone at several spots of the erythema almost cleared it in just 3 days. Chronic (swollen) erythema, in the experience of the writer, requires multiple injections (and bleaching agents).

Peroxide bleaching ointment in the above mentioned rather fresh patient, however, stimulated hyperemia and was therefore omitted. It seems possible that the use of bleaching agents should be restricted to chronic patients only but more experience must be gained for a final conclusion.

\section{Swelling of the face}

In a few patients quick flattening was obtained with both methods but injections of corticosteroid seemed to give quicker results. Although the two methods have remote actions (see under madarosis), when only one half was treated no effect was ever observed on the untreated half.

Special attention is drawn to red swelling of the nose which can be very unsightly, quickly draws concentrated attention and hence is of great psychological influence.

Local subcutaneous injections of corticosteroids make the swelling and redness quickly subside.

Diffuse swelling of the face does not necessarily require multiple injections on different spots. Remote action may render one injection of corticosteroid on each half of the face sufficient (see under madarosis case Dio3/65).

\section{Swollen fingers}

Although Hyfrecator sparking has beneficial slimming effect it takes several weeks or months to complete the treatment.

Quicker results were obtained with corticosteroids which can be injected locally as well as intra-neurally in the supplying nerve ${ }^{2,5}$. 


\section{Madarosis}

It is known that during swelling of the face, eyebrows may fall out and then grow again during remissions. It seems to be caused by pressure on the hair roots due to infiltration. In this chronic symptom both methods seem, in the limited experience of the writer, to be of equal value, likely because it takes the hair root a long time to restore its function. Furthermore diathermic sparking may also stimulate growth of hair via induced hyperaemia.

Remote action of corticosteroid was seen as flattening of the cheeks on the side of the injected eyebrow (see picture case Dio3/65).

Case Dio3/65 : female - 20 years.

- 3 years leprosy of L-type in reaction

- diffuse infiltration of face + ENL on body; madarosis.

After the first infiltration of $5 \mathrm{mg}$. prednisone subcutaneously under the L-madarosis flat-

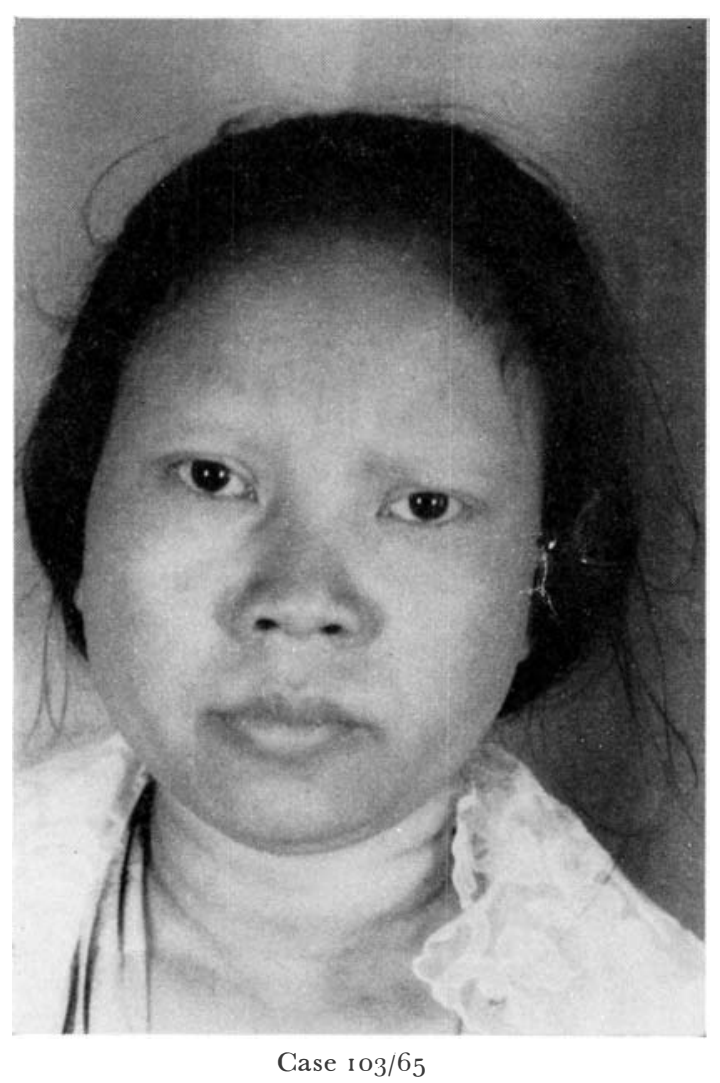

Picture taken after 8 weekly injections. Left corner of mouth lifted due to flattening of left cheek. tening of the L-cheek was seen. Only after 4 months of regular weekly injections was sparse hair observed.

In a leprosarium 35 male and female patients mainly of L-type were at their request ${ }^{2}$ injected weekly with $5 \mathrm{mg}$. cortisone in one madarosis, the other serving as check. After 4 weeks new growth was clearly seen in 15 patients and questionable new eyelorow hair in 2 patients. After the $4^{t h}$ injection, however, further injecting became more difficult in many cases. Injections were abandoned because formation of granulomata due to partly insoluble cortisone was feared (see under discussion). Further evaluation of these preliminary trials can better be obtained with injections of soluble corticosteroids.

\section{Dry scale hyperpigmented skins}

In such cases intraneural prednisolone injections $^{5}$ resulted in visually similar sebaceous

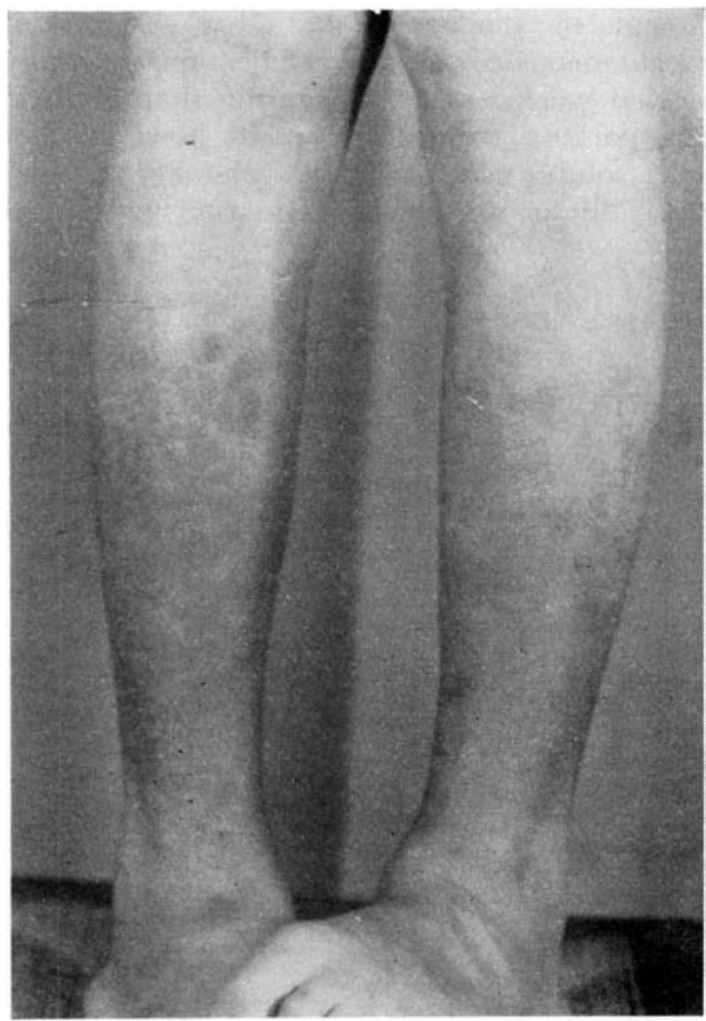

Pat. L.K.S. 
effects as from diathermic sparking. ${ }^{4}$ Due to lack of technical facilities no quantitive measuring of the improvement of the function of the sebaceous glands could be carried out.

As in other cases of dry skin on the extremities equal parts of a lanoline, vaseline and water cream gave good greasing and moisterizing results $^{5}$ (see picture case L.K.S.) Addition of a bleaching agent such as ammoniated mercury is advised.

\section{Elephantiasis}

In one clinically observed patient beneficial effect was seen by intraneural injections in the sciatic nerve. Pain in the foot (? due to röntgenologically determined 'vanished bone'. caused by trophic disturbance) and volume of the elephantiasis decreased. Putting the leg in upward position expedited treatment.

Trials for further evaluation of this therapy will be carried out. ${ }^{2}$

\section{IS G USS I O N}

Except in madarosis and dry scaly skin, subcutaneous corticosteroid injections generally yielded quicker and better results than diathermic sparking. It must be stressed, however, that only soluble corticosteroids should be used especially for madarosis. The writer was forced to utilize the less soluble cortisone-acetate and out of eight out-patients with madarosis, two male patients developed local swelling, in one patient even resulting in the total loss of previous remnants of the eyebrow.

In mass campaigns where the hyfrecator can be used $^{4}$ the author would endorse the use of this instrument for aesthetical purposes as well.

\section{S UMM A R Y}

Attention is drawn to aesthetic management in leprosy, and preliminary results with diathermic sparking and local subcutaneous injections with corticosteroid are compared.

\section{REFERENCES}

I. MUIR, E. 'Manual of leprosy' E. \& S. Livingstone Ltd. Edinburgh (1948).

2. TIO (TIONG HOO), HAN (SIK hIAN) and KUTUT SANTOSO, 'Ambulatory Treatment of Plantar Ulcers'. Lep. Rev. ( 1966$), 37,239$.

3. TIO (TIONG hOO). 'Aesthetic Dermatology in Coloured (Asian) People' (to be published).

4. TiO (TiOng hoo). Lep. Rev ( i 966), 37, 57.

5. TiO (TIONG hoo). Lep Rev. ( I 966, 37, 93.

6. TIO (TIONG HOO). 'Strophulus in Indonesia' (will appear in Dermatologica).

These trials had to be discontinued due to departure of the author. Partially soluble cortisone-acetate had to be used as the supply of soluble prednisolone ran out. 